\title{
Les Mompreneurs
}

Entre entreprise économique, identitaire et parentale

Mompreneurs. In-between Economic Initiative, Identity Quest and Parenting

Crusade

\section{Julie Landour}

\section{OpenEdition \\ Journals}

Édition électronique

URL : http://journals.openedition.org/travailemploi/7536

DOI : 10.4000/travailemploi.7536

ISSN : 1775-416X

Éditeur

DARES - Ministère du Travail

\section{Édition imprimée}

Date de publication : 1 avril 2017

Pagination : $79-100$

ISSN : 0224-4365

Référence électronique

Julie Landour, « Les Mompreneurs », Travail et Emploi [En ligne], 150 | avril-juin 2017, mis en ligne le 11 juillet 2019, consulté le 06 septembre 2019. URL : http://journals.openedition.org/travailemploi/7536 ; DOI : 10.4000/travailemploi.7536 


\title{
Les Mompreneurs
}

\section{Entre entreprise économique, identitaire et parentale}

\author{
Julie Landour
}

\begin{abstract}
Apparues en France à la fin des années 2000, les Mompreneurs se définissent comme des femmes qui créent leur entreprise à l'arrivée d'un enfant, quittant le salariat pour une indépendance qui favoriserait la « conciliation » entre travail et vie de famille. Ce mouvement peut sembler réduit s'il est circonscrit aux seules membres des réseaux « estampillés », mais s'inscrit à la fois dans des processus profonds et transversaux d'individuation, de valorisation publique accrue ces dernières années de l'initiative économique individuelle, et d'accentuation du travail parental, en particulier au sein des classes moyennes et supérieures. S'appuyant sur une enquête de trois ans conduite au sein de l'un des collectifs français de Mompreneurs, cet article propose de restituer les discours de ces femmes en s'intéressant au sens qu'elles donnent à leur entrée dans le non-salariat et en le confrontant aux conditions objectives dans lesquelles elles poursuivent une activité professionnelle indépendante. Après avoir présenté les contours identitaires d'une aventure entrepreneuriale qui se pare des habits de la mère accomplie, on verra comment le cadre familial, et ses éventuelles fluctuations, peut agir sur le parcours de ces femmes initialement bien dotées, révélant la part de fragilité qui se cache sous l'exaltation promise par cette entreprise globale de soi.
\end{abstract}

n 1979 sont mis en place les centres de formalités pour les entreprises (CFE) ${ }^{1}$, l' $^{\prime}$ aide
aux chômeurs créateurs et repreneurs d'entreprise $(\text { Accre })^{2}$ et l'Agence nationale

\footnotetext{
* Centre d'études de l'emploi et du travail (CEET)/Centre Georg Simmel (UMR 8131), École des hautes études en sciences sociales (EHESS); julielandour@msn.com.

1. Interface entre le créateur d'entreprise et les administrations, le CFE est l'interlocuteur permettant d'effectuer les premières démarches pour se mettre à son compte. Pour en savoir plus, voir : https://www.service-public.fr/ professionnels-entreprises/vosdroits/F24023, consulté le 25 août 2017.

2. L'Accre « consiste notamment en une exonération de cotisations de sécurité sociale pendant un an et le maintien des minima sociaux » ; source : http://travail-emploi.gouv.fr/emploi/developpement-de-1-emploi/creation-d-activite/ article/l-aide-aux-chomeurs-createurs-ou-repreneurs-d-une-entreprise-accre, consulté le 25 août 2017.
} 
pour la création d'entreprise $(\text { Ance })^{3}$, autant de mesures et d'institutions destinées à valoriser l'entrepreneuriat pour dynamiser l'emploi et la croissance. Depuis, la volonté des différents gouvernements de promouvoir ces dispositifs d'activation de l'emploi ne s'est pas démentie. Elle s'est même considérablement accélérée au cours des années 2000, avec la promulgation d'une série de lois visant à rendre l'entrepreneuriat plus accessible $^{4}$ (DARBUS, 2008 ; ABDELNOUR, 2013). Sans être directement visées par ces mesures, les femmes en forment une cible à part entière puisqu'elles représentent environ $30 \%$ des travailleur.euse-s non-salarié.e.s ${ }^{5}$. Acteur.rice-s institutionnel-le.s et professionnel-le.s multiplient ainsi les initiatives - chartes, rapports et collectifs ${ }^{6}$ - pour favoriser l'entrepreneuriat féminin.

Le collectif des Mompreneurs est l'une de ces initiatives. Inspiré d'un mouvement qui a émergé dans les années 1990 aux États-Unis, il apparaît en France en 2008. Se définissant comme des femmes qui créent une entreprise à l'arrivée d'un enfant, les Mompreneurs commencent par se regrouper via Internet, en échangeant sur des blogs ou sur le réseau social Facebook, puis se rencontrent dans des groupes informels qui s'organisent bientôt en associations présentes sur tout le territoire français. En se présentant à la fois comme une organisation de formation et de développement d'affaires pour les cheffes d'entreprise et comme des entrepreneuses qui refusent de renoncer à leur vie de famille comme à leurs ambitions professionnelles ${ }^{7}$, elles affichent leur volonté de se positionner comme un groupe professionnel nouveau et semblent subvertir la figure de l'entrepreneur, traditionnellement conjuguée au masculin (AHL, 2004 ${ }^{8}$ ), en y associant la figure saturée de féminin de la mère. Mais cette dernière semble également travaillée par une combinaison inédite, faisant de la maternité un déclic favorisant l'abandon de la stabilité du salariat pour la prise de risque de l'indépendance.

3. L'Ance est devenue en 1996 l'Agence pour la création d'entreprises (APCE) qui a elle-même laissé la place en 2016 à l'Agence France entrepreneur (AFE). Pour découvrir les missions de l'AFE, voir : https://www.afecreation.fr/ pid15040/qu-est-ce-que-l-agence-france-entrepreneur.html, consulté le 25 août 2017.

4. Citons, sans être exhaustive, les lois Dutreil de 2003 (loi pour l'initiative économique) et de 2005 (loi en faveur des petites et moyennes entreprises), ou encore la loi de 2008 de modernisation de l'économie qui instaure le régime de l'auto-entrepreneur.

5. $31,5 \%$ (aides familiales incluses) d'après la dernière enquête Emploi (2016).

6. Citons la « Charte pour l'entrepreneuriat féminin : 20 propositions pour faire avancer l'entrepreneuriat féminin » de janvier 2013 élaborée par le Laboratoire de l'égalité ; le document de travail de Claire Bernard, de Caroline Le Moign et de Jean-Paul Nicolaï d'avril 2013, « L'entrepreneuriat féminin », publié par le Centre d'analyse stratégique ; la Semaine de la sensibilisation des jeunes à l'entrepreneuriat féminin lancée en mai 2013 par le ministère des Droits des femmes avec l'APCE et la floraison des réseaux de femmes cheffes d'entreprise, sur lesquels Marion RABIER (2013) a réalisé sa thèse de sociologie.

7. Site internet de l'association étudiée, consulté en janvier 2014. Pour des raisons de confidentialité et de préservation du terrain, l'adresse précise du site ne peut être délivrée et les formules initiales ont été modifiées pour limiter toute identification.

8. Helen AHL produit une stimulante analyse des travaux portant sur les « entrepreneurs » : en revenant d'abord sur les travaux des économistes et des chercheurs en management, elle rend compte de l'implicite masculin qui entoure la description de l'entrepreneur. En se déportant ensuite sur les travaux, principalement conduits en sciences de gestion, consacrés plus spécifiquement aux femmes entrepreneuses, elle fait état des mécanismes de reconstruction des rapports sociaux de sexe qui irriguent ces recherches. 
En nous appuyant sur une enquête de trois ans conduite dans l'un des collectifs français de Mompreneurs (encadré), nous proposons d'étudier dans une optique panoramique et matérialiste ${ }^{9}$ cette forme féminisée de travail non salarié faisant volontiers de l'indépendance la voie idéale pour articuler travail et famille. Il s'agit de restituer les discours des Mompreneurs en s'intéressant au sens qu' elles donnent à leur entrée dans le non-salariat, tout en les confrontant aux conditions objectives dans lesquelles elles poursuivent une activité professionnelle non salariée.

Dans un premier temps, est ainsi exposée la source de valorisation que présente la création d'une activité indépendante pour ces anciennes salariées qui se considéraient empêchées dans leur engagement dans le travail : déçues ou exclues du salariat, elles endossent non seulement les habits de l'aventure entrepreneuriale ainsi que ceux de la mère accomplie. Les deuxième et troisième temps de cet article mettent cette dimension identitaire à l'épreuve de leurs situations d'abord professionnelle puis, plus largement, socio-économique : plusieurs cas nous permettront de voir comment, dans un cadre familial stable, l'activité professionnelle de ces femmes est marginalisée par un travail

\section{ENCADRÉ}

\section{Méthodologie de l'enquête}

Cet article mobilise une recherche conduite entre 2011 et 2014 au sein de l'une des associations de Mompreneurs françaises. Ces dernières sont apparues en France à la fin des années 2000 (cf. supra), parallèlement à la création du régime de l'auto-entrepreneur. Deux organisations distinctes ont été fondées par deux femmes aux propriétés sociales favorisées, quoique hétérogènes. Très vite, ces deux associations sont entrées en concurrence, l'une défendant une activité de lobbying et l'autre d'entraide entre ses membres (LANDOUR, 2015a). Nous étudions cette dernière : comptant au moment de l'enquête près de 500 membres répartis dans vingt antennes régionales, c'est, d'une part, l'association de Mompreneurs qui s'est le plus largement implantée en France et, d'autre part, l'un des réseaux de femmes entrepreneuses les plus importants numériquement en France.

La démarche de recherche adoptée combine de multiples approches méthodologiques : analyse documentaire, observations de différents types de rassemblements (convention annuelle, réunion mensuelle des antennes, etc.), suivi des profils des membres et des échanges tenus sur Facebook, entretiens semi-directifs, enquête quantitative, etc. Sont plus particulièrement utilisés ici les 54 entretiens semi-directifs réalisés auprès de membres de ce collectif de Mompreneurs en Île-de-France $(n=37)$ et dans l'Hérault $(\mathrm{n}=17)$ et l'enquête quantitative en ligne conduite auprès de l'ensemble des membres de l'association du 17 septembre au 6 octobre 2013, à partir de son fichier d'adhérentes. Sur 417 femmes, 268 ont répondu à l'intégralité du questionnaire, soit un taux de retour de $64 \%$ avec un échantillon proche du profil de l'ensemble des adhérentes.

9. À la suite notamment de Christine DelPHY (1998, 2015), j'entends par « matérialisme » l'intérêt porté aux conditions matérielles de réalisation d'un fait social, et notamment aux structures économiques et sociales dans lesquelles elles s'insèrent. 
domestique et parental intense, dans lequel elles s'engagent fortement tout autant qu'elles y sont (ré)assignées. Pour conclure, nous examinerons plus attentivement les situations de celles dont le cadre familial évolue, notamment en cas de dissolution de leur couple : c'est alors que le déclassement individuel objectif de ces femmes pourtant bien dotées apparaît, révélant la part de fragilité qui se cache sous l'exaltation promise par une entreprise qui n'est pas seulement économique, mais touche jusqu'à la part la plus intime de ces parcours.

\section{S’accomplir dans l'indépendance}

« Nous avons décidé de créer notre activité pour gagner en liberté et, surtout, équilibrer nos vies personnelle et professionnelle » : c'est ainsi que le site internet de l'association étudiée présente «l'état d'esprit » des Mompreneurs. Cette rhétorique de l'individu maître de son destin est particulièrement présente dans les discours des femmes rencontrées au cours de l'enquête. S'il convient de la discuter, il s'agit également de ne pas l'occulter : elle rend en effet compte du sens que les individus donnent à leurs actions et participe de l'analyse de leur positionnement. Ainsi, ce premier temps propose de revenir sur les discours que les Mompreneurs tiennent sur cette trajectoire professionnelle de sortie du salariat pour l'indépendance, en déployant d'abord leur critique féminine du salariat, puis le sens qu'elles donnent à l'exercice d'une activité professionnelle indépendante.

\section{Une critique féminine du salariat}

Si les Mompreneurs présentent leur entrée dans l'indépendance comme un choix volontaire, leur bifurcation fait pourtant suite à une inscription altérée dans le salariat, que les résultats de notre enquête quantitative révèlent : parmi les répondantes, on compte $28 \%$ de femmes qui étaient au chômage avant de devenir Mompreneurs, dont la moitié de chômeuses de longue durée, ce qui représente une proportion nettement supérieure à celle observée auprès de l'ensemble des femmes de 25 à 49 ans $(9,1 \%$ en $2015^{10}$ ). Par ailleurs, $8 \%$ étaient sans activité professionnelle et $2 \%$ se déclaraient étudiantes. De plus, celles qui avaient un contrat de travail salarié n'étaient pas systématiquement en poste : $65 \%$ l'étaient, de retour de congé maternité ou de congé parental ; en revanche, $9 \%$ étaient encore en congé maternité et $15 \%$ en congé parental $^{11}$. Enfin, parmi les 205 femmes qui se sont effectivement mises à leur compte

10. Source : Insee, enquête Emploi 2015.

11. En 2010, $28 \%$ des mères d'enfants de moins de 8 ans ont interrompu leur activité au moins un mois en prenant un congé parental à temps plein, tandis que $21 \%$ travaillent à temps partiel (source : enquête Emploi, module complémentaire sur la conciliation entre vie familiale et vie professionnelle 2010). Si ces chiffres ne correspondent pas exactement à ceux que nous avons obtenus dans notre enquête, ils nous incitent à penser que le congé parental est moins souvent pris par les Mompreneurs que par l'ensemble des femmes. 
après avoir occupé un emploi salarié, $24(11 \%)$ arrivaient au terme d'un contrat précaire (CDD, intérim, etc.) et 96 (47\%) avaient signé une rupture conventionnelle ${ }^{12}$.

Au final, sur l'ensemble des répondantes, seules $40 \%$ des Mompreneurs étaient en poste avant de créer leur activité. Si ce constat fait écho aux difficultés rencontrées par les femmes pour s'ancrer dans le marché du travail (MARUANI, MERON, 2012), en particulier après une ou plusieurs maternités (MeURs et al., 2010 ; PAILHÉ, Solaz, 2006, 2012 ; RÉGNIER-LOILIER, 2009), il convient de l'examiner plus en détail à la lueur des propriétés sociales des Mompreneurs. Ces dernières constituent en effet un groupe certes hétérogène mais globalement favorisé : selon les catégories de notre enquête, $55 \%$ des répondantes déclarent que dans leur emploi précédent, elles faisaient partie des catégories supérieures (ingénieure, cadre, directrice ou indépendante ${ }^{13}$ ) ; $23 \%$ affirment plutôt relever des classes moyennes (agent de maitrise, technicienne ou assimilée) et $22 \%$ des milieux plus populaires (ouvrière ou assimilée, employée de bureau, de commerce ou personnel de services). Au sein des Mompreneurs, on compterait ainsi trois fois plus de membres des catégories supérieures que dans l'ensemble de la population féminine nationale (17\% d'après le recensement de 2008). Ajoutons que cette situation favorable est renforcée par leur assise conjugale : $86 \%$ vivent en couple et $77 \%$ sont mariées ${ }^{14}$.

La relative variété des positionnements introduit des nuances dans l'altération de la relation de ces femmes au salariat. Certaines n'ont jamais réussi à s'y inscrire de façon pérenne et satisfaisante : c'est le cas de Nathalie, fille d'un ouvrier spécialisé et d'une mère sans profession qui connaît un « destin pas très sympa » lorsque son mari la quitte. Nathalie, qui cherche à se démarquer de son milieu familial par un très fort attachement au domaine des arts et des lettres, s'engage dans un cursus universitaire littéraire où, peu armée pour réussir, elle « rate le coche des études » dès la première année. Elle enchaîne alors les emplois précaires, se marie jeune et divorce rapidement tout en entamant un cursus professionnalisant dans la communication. Diplômée d'un brevet de technicien supérieur (BTS) et montée à Paris, elle s'investit dans plusieurs projets culturels qui la passionnent mais qui ne lui permettent pas d'obtenir une situation stable malgré tous les efforts déployés. À 30 ans, « épuisée moralement et physiquement » et alors qu'elle envisage avec son second mari, cadre de l'informatique, d'avoir un enfant, elle renonce au salariat pour créer son activité indépendante :

« Je voulais changer ma vie. Donc changer ma vie, c'était sans doute avoir un enfant, changer d'orientation professionnelle, ne plus être de la chair à canon. Comme ça [c'est-à-dire, avec sa situation avant de changer de vie], je voyais que je pouvais pas me faire recruter, j'avais pas de diplôme, je voulais pas retourner à l'école,

12. Le caractère pleinement consenti et favorable aux salariés de ce type de rupture a été remis en cause dès ses premières évaluations (BERTA et al., 2012 ; DALMASso et al., 2012).

13. Selon les modalités proposées dans notre enquête.

14. Pour plus de détails, voir LANDOUR, 2015a. 
je voulais que ce système s'arrête, et là, je me suis dit, ma chance, c'est peut-être l'indépendance. »

(Nathalie, 38 ans, société de services aux entreprises, entretiens du 24 juillet et du 6 août 2013)

D'autres, issues de milieux plus favorisés et davantage diplômées, s'insèrent sans difficultés et occupent un emploi salarié stable (en contrat à durée indéterminée [CDI]), souvent avec une position de cadre, de profession intermédiaire ou d'employée. Ce sont alors des maux éprouvés dans leur quotidien de travail qui ne leur permettent pas de tenir, maux qui se déclinent selon deux axes. Il arrive que l'entreprise connaisse des difficultés économiques et ne soit plus en mesure de garantir un emploi par ailleurs satisfaisant : c'est ce qu'a par exemple vécu Florence, cadre polyvalente et autodidacte dans une petite entreprise de la région parisienne. Lorsqu'elle est licenciée pour raisons économiques, elle a trois enfants avec Hugues, son mari, dont la carrière professionnelle est plus discontinue. Elle raconte :

«Après avoir été licenciée, [...] euh, je me suis dit : "Bon qu'est-ce que je fais ?" J'ai pensé à faire une VAE [validation des acquis de l'expérience], ouais, je crois que c'est ça, et Hugues depuis plusieurs années me disait : "Florence, monte ton entreprise, monte ton entreprise !"Et du coup, je me suis dit : "Après tout, pourquoi pas ?”Mon employeur me demandait lui aussi de monter mon entreprise pour pouvoir me faire travailler, [...] donc du coup, j'ai décidé de me mettre à mon compte. »

(Florence, 40 ans, société de services aux entreprises, entretien du 10 avril 2012)

Dans des cas un peu plus nombreux au sein de la population enquêtée, ce sont les femmes elles-mêmes, souvent déjà mères, qui ne tiennent plus le rythme de travail lié à leur statut de cadre. Plus fréquemment employées dans des entreprises de taille importante, elles sont en outre confrontées à des organisations du travail dont elles ne perçoivent plus le sens et à des pressions que leur corps et leur esprit ne sont plus en mesure d'absorber. C'est ce que décrit Rebecca, cadre supérieure d'une grande entreprise de cosmétiques, mariée à un autre cadre supérieur, par ailleurs déjà père de deux enfants. Le couple a trois enfants en commun. Cette femme, issue de la petite bourgeoisie francilienne et titulaire d'un master en droit social, se heurte à la compétition propre à ce type d'organisation, dont elle remarque qu'elle s'exacerbe à chacune de ses grossesses, jusqu'à ce que son corps lâche et rende son maintien en poste impossible (elle est arrêtée plusieurs mois et finit par négocier un licenciement) :

« Il y a une réunion hyper houleuse avec la fille qui était en train de me piquer mes dossiers [...]. Je la remets à sa place, elle s'en plaint, on fait une réunion avec notre directrice marketing commune, où elle est odieuse avec moi : elle sort que des horreurs, je la renvoie dans ses vingt-deux [mètres] et je me défends et on rentre dans notre bureau et là, elle redevient mais tout chou, tout crème, comme elle avait toujours été jusque-là parce qu'elle avait très bien caché son jeu, c'est-à-dire : "Mais alors, tes 
vacances, ça s'est bien passé ? [elle prend une voix mielleuse]", alors que l'on venait de s'arracher la tête quoi ! Un truc hallucinant, et là je bugge. J'ai fait un burn-out. »

(Rebecca, 36 ans, société de services aux entreprises, entretien du 24 octobre 2012)

D'autres enfin sont insérées de façon satisfaisante (en CDI, dans des postes qui ne leur posent pas de difficultés particulières), mais ne trouvent plus dans ce type d'emploi les leviers nécessaires à leur réalisation personnelle. Elles envisagent de construire ailleurs le cadre de leur accomplissement, dans un projet qui englobe toutes les sphères de leur vie. C'est le cas de Sonia, secrétaire de direction d'une très grande entreprise du secteur des télécommunications, mariée avec un agent d'une entreprise publique avec lequel elle a deux enfants. Sonia est dans l'ensemble contente de son poste et de ses relations de travail, mais souhaite progresser au sein de l'entreprise ; pour cela, elle suit plusieurs formations qualifiantes destinées à accompagner son ascension professionnelle. Malgré ses demandes et l'appui du directeur avec lequel elle travaille, « on lui fait comprendre qu'elle ne pourra jamais évoluer ». Sa mère, ancienne couturière divorcée, vit alors dans une grande précarité et Sonia envisage de monter un projet professionnel lui permettant d'exercer les responsabilités professionnelles auxquelles elle aspire tout en créant un « emploi sur-mesure » à sa mère. Pour lancer son activité, elle se saisit d'un plan de départs volontaires mis en place au sein de l'entreprise.

On voit donc apparaître derrière l'ensemble de ces parcours une relation globalement altérée au salariat, et qui prend différentes tournures selon les propriétés sociales initiales de ces femmes (qualifications, âge, etc.), les caractéristiques de leur insertion dans le salariat (type d'entreprise et santé économique de celle-ci, poste occupé, etc.) et leur situation conjugale et familiale. Derrière ces parcours se dessine également une critique du salariat, qui ne représente plus à leurs yeux une sphère d'affiliation enviable. Cette critique est toutefois plus féminine que féministe : si les difficultés qu'elles rencontrent sont largement liées à leur position infériorisée dans les rapports sociaux de sexe (poids des qualifications dans l'insertion, ségrégation horizontale et verticale, charge productive et reproductive, etc.), les femmes interrogées en délivrent un récit personnalisé qui ne leur permet pas d'élaborer une lecture plus politique de leur situation $^{15}$. Cette prime laissée à l'individu se prolonge d'ailleurs dans les perspectives dans lesquelles elles se projettent en créant leur entreprise.

\section{Le travail indépendant comme épanouissement du soi}

À la suite des difficultés éprouvées dans le salariat, il arrive que les femmes enquêtées traversent des « trous » d'air dans leur parcours, en se retirant plus ou moins longtemps de l'activité professionnelle. Largement positionnées dans les franges les

15. Ajoutons sur ce point que le terme «Mompreneurs » et sa féminisation incomplète n'ont jamais été mis en débat. Plus largement, nous avons observé une grande ambivalence dans les discours au sujet du féminisme, les pratiques observées renvoyant en outre à une imprégnation forte des rôles sexués. Pour plus de détails, nous renvoyons à LANDOUR, 2015b. 
mieux dotées de la société française, elles considèrent toutefois le travail comme un vecteur identitaire extrêmement fort : leurs discours font régulièrement allusion à la figure repoussoir de la femme au foyer, tandis que le chômage de longue durée y est implicitement assimilé à une forme d'assistanat également critiquée. Cette focalisation sur la dimension identitaire de l'activité professionnelle est renforcée par une grande proximité avec le monde de l'indépendance : $72 \%$ des Mompreneurs reconnaissent avoir une ou plusieurs personnes indépendantes dans leur entourage. Plus précisément, on constate une surreprésentation des parents indépendants (près de deux fois plus nombreux que dans la population générale pour les pères et trois fois pour les mères), ainsi que des conjoints (16\% sont artisans, commerçants ou chefs d'entreprise, contre $8 \%$ dans la population active masculine $)^{16}$. Ces femmes bénéficient ainsi d'un « bain entrepreneurial », structuré en habitus pour celles qui cumulent une parentèle et un conjoint indépendants. Cette socialisation contribue à faire de la création d'une activité indépendante un moyen valorisant d'exercer un travail et de s'affranchir du salariat critiqué pour son caractère parfois éprouvant. Par ailleurs, les enquêtées passent largement sous silence les protections qui en découlent, quand elles ne les remettent pas en cause (cas de la retraite notamment). Dans l'enquête quantitative, $56 \%$ disent avoir créé une entreprise pour exercer une activité qui corresponde à leurs valeurs ; $24 \%$ envisagent l'indépendance comme une façon de pratiquer le métier de leur rêve. Les activités qu'elles choisissent traduisent autant de formes idéalisées d'accomplissement de soi.

Certaines cherchent ainsi à renouer avec une forme sublimée du métier (DUJARIER, 2006), mêlant corps, pensée et émotion. Les métiers artisanaux sont particulièrement investis (OsTY, 2003), qu'ils soient réellement exercés ou non. Outre qu'ils nécessitent une virtuosité extrême pour être exercés au mieux, ils renouent avec des qualités féminines essentialisées, comme la minutie, l'organisation ou le goût (notamment dans le cas de la mode, qu'elle soit vestimentaire, décorative ou encore liée aux dernières tendances gastronomiques), comme le montre l'exemple de Laurence : ancienne employée de commerce mariée à un agent de police et mère d'un enfant, Laurence fabrique ses propres bijoux depuis de longues années. Au moment de son licenciement, elle lance son activité et une marque pour commercialiser les créations qu'elle confectionne à la main avec «passion » et « enthousiasme », affichant un très grand « plaisir » à chercher des matières premières chez les différents fournisseurs, à inventer de nouveaux modèles et à travailler sur les matériaux et objets ${ }^{17}$.

Une autre des caractéristiques des activités choisies par les Mompreneurs consiste à apporter de l'aide à autrui ${ }^{18}$, mais à un autrui qui ne serait toutefois pas n'importe lequel. Aucune n'a ainsi bifurqué vers le secteur sanitaire ou social, et aucune ne s'investit dans des associations caritatives. Le cas de Frédérique est exemplaire de cette volonté d'aider des « autres semblables » : fille d'instituteurs mariée à un cadre informatique, Frédérique est employée administrative d'une petite entreprise dans le

16. Pour plus de détails, voir LANDOUR, 2015a.

17. Cf. sur cette question l'article d'Anne JouRdaIN (2017) dans ce numéro, qui présente la situation des artisanes d'art.

18. Encore une caractéristique essentialisée comme féminine. 
sud de la France, dont elle est licenciée économique pendant le congé parental de trois ans qu'elle prend à la naissance de son second enfant. Au terme de ce congé, durant lequel elle a particulièrement apprécié le temps passé auprès de ses enfants, elle décide de créer une activité lui permettant de rester à domicile. Elle puise alors dans ses goûts d'adolescente : « Le truc que j'avais depuis très longtemps, c'était de mettre par écrit les souvenirs des gens, des particuliers, des mémoires familiales et ça, depuis que j'ai 15 ans quoi, c'était vraiment un truc. » Elle décide de devenir écrivain public, mais ne se tourne pas vers le secteur de la justice et du social qu'elle perçoit comme aride et qui la rebute : «Voilà, des choses comme la justice, des choses un peu..., soulever des problèmes et tout, bon, c'est pas trop mon truc, je suis pas trop..., enfin, voilà, y a des choses tu les sens ou pas. » Ces femmes s'orientent donc volontiers vers des activités que l'on pourrait ranger parmi celles du care, mais un care que nous qualifions de «propre » dont bénéficient des personnes qui ne sont pas dans des situations trop éloignées des leurs (autrement dit, qui ne sont par exemple ni malades, ni en grande précarité économique ou sociale).

C'est aussi la mise en avant de soi, parfois totalement, qui prime dans l'activité qu'elles ont créée, certaines n'hésitant pas à faire de ce qu'elles sont une prestation à part entière. Elles donnent ainsi très souvent leur propre nom à leur activité. Mélanie, fille d'un pharmacien et d'une professeure d'espagnol, qui affirme avoir toujours refusé les «CDI-cadenas » qu'elle a par ailleurs eu les plus grandes difficultés à obtenir après son master 2 en identité visuelle, présente une version paroxystique de l'accomplissement identitaire permis par l'exercice d'une activité indépendante. Elle a par exemple appelé sa société de «mise en valeurs », «Richesse de soi » : elle propose d'accompagner des indépendants dans leur communication, à travers des formations collectives et des séances de coaching individuel visant la «mise en cohérence » de la personnalité et de l'activité. Chez Mélanie, la mise en scène de soi va plus loin : licenciée après des relations houleuses avec sa patronne, elle décide de créer son activité. C'est alors que son compagnon meurt d'un cancer foudroyant. Si elle affiche une certaine discrétion à ce sujet, elle fait régulièrement état à travers les différents posts de son blog de la résilience dont elle a su faire preuve suite à cet événement tragique. Elle en fait même un gage de la qualité des conseils qu'elle vend aux indépendants qu'elle accompagne... dont bon nombre sont des Mompreneurs :

«Pendant un long moment, je me suis dit que c'était "normal" : la vie avait été assez dure avec moi pour qu' au moins, dans mon entreprise, je sois sereine. Mais non, rien n'est "normal". Et cela n'a pas rien à voir avec la bonne étoile, ni l'optimisme, ni l'estime de soi. Dans ce monde où il est de bon ton de dire que la crise est partout, mon business et ma vie vont vraiment bien. Même quand ma vie était un enfer, mon entreprise se développait bien. Pourquoi, et pourquoi moi ? On m'a dit il y a peu : "C'est incroyable, avec toutes les catastrophes que tu as traversées, tu arrives toujours à les transformer pour les orienter dans ta direction." Sûrement parce que je ne pense pas en termes de business plan, de cibles, d'obstacles ni de difficultés à 
résoudre. D'ailleurs ce sont des mots qui n'appartiennent pas à ma façon de parler. Ça ne signifie pas que ça n'existe pas. Ça veut dire que je regarde différemment. »

(Extrait du blog dans lequel Mélanie présente son activité, publié le 9 avril 2015)

Le travail qu'elles accomplissent avec le statut d'indépendante est ainsi avant tout pensé comme un vecteur d'accomplissement identitaire. Le changement d'orientation professionnelle des Mompreneurs évoque, à bien des égards, les reconversions analysées par Pierre BouRdIEU au sein de la «petite bourgeoisie nouvelle » (1979, pp. 409-421), et notamment auprès de ses membres féminins. Elles s'orientent vers de nouvelles professions, à la lisière du privé et du public, dans lesquelles l'expression et la présentation de soi sont des éléments clés de l'activité. Si les Mompreneurs sont le plus souvent davantage diplômées que leurs parents (par effet de massification scolaire) et davantage situées du côté du pôle économique que les femmes étudiées par P. Bourdieu, elles ont en commun une bonne volonté culturelle, une éthique domestique et un enthousiasme pour la consommation. Le fait d'entreprendre - au sens de créer une activité - constitue à leurs yeux un élément qui les distingue, favorisant une plus grande expression de soi et de son « authenticité », et une prise de risque plus valorisante qu'un salariat qu'elles considèrent souvent comme anesthésiant. Chez les Mompreneurs, les apports instrumentaux du travail (rémunération, diverses protections sociales, etc.) sont d'autant plus mis de côté qu'ils sont masqués à la fois par une vision psychologisée des rapports sociaux et une assise conjugale qui dilue l'autonomisation des ressources et les apports respectifs des conjoints au sein du ménage. Cette situation familiale doit être particulièrement prise en compte dans l'analyse du parcours des Mompreneurs, non seulement parce que les activités indépendantes sont de fait des structures économico-familiales, mais aussi parce que le travail parental en est partie prenante.

\section{Des engagements professionnels, parentaux et domestiques en concurrence}

Par leur dénomination, les Mompreneurs affirment leur volonté de mettre sur un pied d'égalité travail et maternité, engagement professionnel et engagement parental ${ }^{19}$.

\footnotetext{
19. Nous préférons utiliser le terme parental plutôt que maternel, partant de l'idée que l'ensemble des tâches regroupées sous le terme de parentalité peuvent être indifféremment accomplies par une femme ou un homme (en dehors du travail génésique lié à la grossesse et de l'accouchement lui-même). Le fait que ces tâches soient effectuées plutôt par la mère ou plutôt par le père reste à faire émerger de l'enquête et doit être analysé comme un fait social à part entière. Nous entendons donc par travail parental l'ensemble des tâches réalisées par les adultes autour de leur enfant. Nous y intégrons le travail de santé (suivi alimentaire, sanitaire, etc.), mais aussi éducatif (par exemple, apprentissage des règles de politesse), scolaire (suivi des devoirs) et tout ce qui, concrètement mais également mentalement, vient alimenter la prise en charge des enfants. Le travail parental évolue au fil des différentes étapes de la vie marquées par des seuils et des espaces institutionnalisés (entrée à l'école et ses différents rythmes), et par des événements plus
} 
Comprendre le phénomène implique donc de s'intéresser de près à ce que recouvre cette mise en avant de l'engagement parental et plus particulièrement de la maternité, ce qui rend la catégorie inédite malgré une existence de fait ancienne des mères à la tête d'activités indépendantes. Le premier temps de cette partie s'attache à cerner la manière dont, chez les Mompreneurs, cet engagement parental s'incarne dans des discours et des actes. Le second temps dessine la dynamique de réassignation à la sphère domestique qui en découle.

\section{Une conception exigeante de la parentalité}

Entretiens et observations ont permis de collecter des éléments de natures diverses sur les pratiques parentales effectivement mises en place par les Mompreneurs. Portées par une volonté initiale d'affirmer le maintien de leur inscription professionnelle, elles ne placent pas la maternité au cœur de leur discours alors qu'elle occupe une place déterminante dans leurs parcours. La surreprésentation parmi les Mompreneurs de femmes qui ont eu des difficultés à devenir mères met en lumière ce phénomène. Carole, diplômée d'un master en droit de l'immobilier et mariée à un cadre informatique, a adopté un enfant. Si elle s'efforce au cours de l'entretien de mettre en avant son engagement professionnel, elle reconnaît toutefois que l'adoption a été un "parcours du combattant », qui a pesé sur l'ensemble de sa biographie, jusqu'à entraîner sa démission. Après avoir accumulé les congés sans solde au cours de la procédure, puis un congé parental à l'arrivée de son enfant quatre ans plus tard, elle ne supporte plus de ne « voir sa fille que vingt minutes par jour ». La disponibilité des parents - et tout particulièrement des mères - est de fait au cœur de la définition du travail parental de ces femmes (LANDOUR, 2016).

Au-delà du seul discours, cette injonction à la disponibilité à laquelle les Mompreneurs souscrivent pèse concrètement sur leurs parcours. Au moment de la petite enfance par exemple, les divers soins à apporter à l'enfant requièrent une plus ou moins grande disponibilité physique et mentale. Les tâches les plus chronophages sont régulièrement citées par les Mompreneurs : accouchement à domicile, portage proximal en écharpe, partage de lit avec l'enfant, allaitement au sein prolongé (parfois jusqu'à deux ans) et autres pratiques d'alimentation et de soins (couches lavables, nourriture faite maison, etc.). Plus tard, la présence auprès des enfants, notamment autour de l'école, peut être également particulièrement prenante. C'est ce qu'illustre l'exemple de Frédérique évoqué plus haut. Malgré son licenciement, celle-ci dit s'être « régalée » pendant son congé parental, ce qui lui a permis de faire déjeuner ses enfants le midi, de garder le plus jeune à la maison les après-midi ou de s'investir au sein de l'association des parents d'élèves. Tout en établissant au cours de l'entretien la liste des

ou moins prévisibles (évolution de la configuration familiale, etc.). Nous ajoutons que le travail parental peut aussi être effectué par d'autres adultes que les parents (d'autres membres de la famille par exemple, ou des professionnels comme les assistantes maternelles ou les auxiliaires de puériculture). 
activités qu'elle a mises en place pour ses enfants, elle signale les cas de mères qui, de leur côté, semblent s'exonérer d'un tel niveau d'engagement auprès de leurs enfants :

« Tu as des mamans au foyer qui tournent en rond aussi, il faut le reconnaître. Moi, j'ai une copine qui, elle, est au foyer mais elle compte les jours des vacances. Elle te dit : "Encore une semaine à tenir." Là, tu te dis, bon, quelque part, bon voilà, elle est bien au foyer mais en même temps, des fois, ses gamins, ils la saoulent. En plus elle est pas du tout balades, elle est pas du tout sorties, donc ils ont vite fait de tourner en rond et quand ils tournent en rond... »

(Frédérique, 39 ans, auto-entreprise de services aux particuliers, entretien du 11 juin 2013)

Frédérique condamne l'oisiveté de certaines mères au foyer, dont elle s'efforce de se distinguer, mais critique également les mères restées actives qui « courent tout le temps » pour « un salaire pas mirobolant », blâmant leur manque de présence auprès de leurs enfants. C'est ce plaisir pris auprès de ses enfants - qui lui permet aussi sans doute de se distinguer facilement des autres mères - qui l'a incitée à reprendre une activité indépendante à la fois valorisante et adaptable à la présence, importante et dense, qu'elle souhaite assurer auprès de ses enfants :

« C'est le côté, j' ai une activité professionnelle à développer parce que c'est mon truc qui me sort des tripes, c'est mon envie. Voilà, j' ai vraiment envie de tout faire pour que ça marche et ça va marcher parce que j'y crois tellement fort que ça ne peut pas être autrement, et en même temps j'ai des enfants, et je veux arriver à tout cumuler, je veux arriver à tout : que mes enfants ne pâtissent pas de mes absences, que je sois là pour eux parce que j'aime ça, que j'aille les chercher à midi parce que j'aime les avoir avec moi le midi, parce que j'aime être à l'école à cinq heures, j'aime assister aux spectacles. Voilà j'aime ça et je suis tout ça en fait. Je suis une maman présente et je suis une nana qui crée son activité professionnelle quoi ! »

(Frédérique, 39 ans, auto-entreprise de services aux particuliers, entretien du 11 juin 2013)

Parallèlement à la création d'une entreprise économique, dont on a vu quelle était également identitaire, les Mompreneurs conduisent également une entreprise parentale, à travers leur engagement dans un travail parental intense. Si ce dernier est structuré par des activités puérocentristes (GARCIA, 2011), ces femmes s'en saisissent pour se distinguer des autres mères (LANDOUR, 2016). C'est ainsi d'une entreprise globale de soi (EHRENBERG, 1991) que procèdent ces parcours, le revers salarial semblant gommé par une initiative individuelle qui ne concerne plus seulement le travail, mais irrigue les sphères familiale et, plus largement, personnelle. L'initiative n'entraîne toutefois pas nécessairement une pleine réussite sociale. Notamment, le double engagement des Mompreneurs a une incidence très concrète sur leurs parcours, pour qui chausse des lunettes matérialistes : il enclenche une assignation accrue de ces femmes au sein de la sphère domestique. 


\section{Une assignation globale à la sphère domestique}

S'engageant dans une activité indépendante, avant tout source d'accomplissement, les Mompreneurs déploient parallèlement une maternité intensive (HAYs, 1996), concrétisée à travers un maternage étroit devenu lui-même une source de positionnement social. S'il va de soi que les activités parentales, bien que visibles hors du foyer, sont accomplies avant tout dans la sphère privée, notre enquête révèle que $74 \%$ des Mompreneurs exercent leur activité professionnelle à domicile. Cette installation au domicile doit être reliée à la faiblesse des investissements initiaux : parmi les $66 \%$ de Mompreneurs qui disent avoir investi moins de 8000 euros, $45 \%$ ont investi moins de 2000 euros et $48 \%$ de 2000 à 4000 euros. En mobilisant avant tout leurs ressources personnelles et familiales pour créer leur activité (seules $20 \%$ disent avoir sollicité un prêt, et seulement $11 \%$ un prêt à la banque), les Mompreneurs disposent de faibles marges de manœuvre économiques, ce qui ne leur permet pas d'investir dans des locaux autres que leur domicile pour exercer leur activité.

Les Mompreneurs tendent à valoriser leurs conditions de travail qui leur permettent de travailler tout en surveillant leurs enfants, en particulier lorsqu'ils sont petits et réputés dormir tout le temps. Dans les faits, plusieurs indiquent qu'il leur est impossible de s'astreindre à une véritable discipline de travail quand les enfants sont présents - les pères étant le plus souvent absents dans la journée. C'est ce qu'explique par exemple Lili. Cette graphiste, qui « adorait la vie en entreprise », se voit imposer une rupture conventionnelle peu avant de tomber enceinte et de déménager dans la très grande couronne francilienne avec son mari, gérant d'un magasin de vêtements. Des missions ponctuelles que lui confie son ancien employeur la conduisent à devenir auto-entrepreneuse pendant sa grossesse, cette mise à son compte se pérennisant « un peu au hasard », au gré des missions qui se présentent à elle. Après la naissance, elle essaie de travailler tout en gardant son bébé. Elle a toutefois des difficultés à tout mener de front, tant le fait de ne pas s'occuper de la petite alors qu'elle est présente à ses côtés contrevient à la manière dont elle entend élever son enfant :

«L'année dernière, c'était vraiment horrible. Donc j'avais fait de la compta, et elle est restée une fois une heure et demie dans son parc toute seule : alors elle jouait mais moi, je veux pas avoir un enfant légume, je veux pas le laisser tout seul s'asseoir dans son parc. L'intérêt, c'est de faire de la peinture avec elle, bon parce que j'aime ça, mais plein d'autres choses, on joue, on lit, on chante des chansons, et j'ai envie qu' elle s'éveille et je vais pas la mettre devant la télé quoi, ça m'intéresse pas du tout. »

(Lili, 32 ans, auto-entreprise de services aux entreprises, entretien du 23 juin 2012)

Comme Frédérique plus haut, Lili est attentive à la stimulation de son enfant, ce qui doit passer par une présence permanente favorisant la démultiplication des activités d'éveil. Elle est donc dans l'incapacité de tenir ses exigences parentales tout en travaillant et doit se résoudre à faire garder sa fille par une «nounou », d'abord à mi-temps, puis à temps plein lorsqu'elle est contrainte d'exercer son activité également 
à temps plein alors que la petite a cinq mois ${ }^{20}$. Mais son temps plein n'en est pas vraiment un : son conjoint garde en effet la petite le mercredi et Lili explique avoir les plus grandes difficultés à travailler quand la famille est réunie. Outre le travail parental, Lili est en grande partie responsable du travail domestique : si son compagnon est plutôt en charge des courses, c'est elle qui assure les lessives (importantes avec un enfant en bas âge), le rangement et les autres tâches ménagères. Lorsqu'elle a demandé davantage d'aide à son compagnon, celui-ci lui a proposé de prendre les services d'une femme de ménage deux heures par semaine. Mais Lili culpabilise et veille à ce que leur appartement soit rangé lorsque arrive leur femme de ménage :

« Ça a l'air de rien comme ça parce qu'elle fait le ménage que deux heures, mais pour qu'elle fasse le ménage il faut que ça soit rangé, parce qu'elle vient pas pour ranger. Donc t'es obligée de faire en sorte que ça soit plus ou moins rangé, donc de garder une constance au fur et à mesure. Et finalement quand elle vient, c'est vraiment que pour faire le ménage, alors que moi quand je fais le ménage, je range en même temps. »

(Lili, 32 ans, auto-entreprise de services aux entreprises, entretien du 23 juin 2012)

Soulignons également que, contrairement à ce qu'induit l'idée de conciliation, la superposition des sphères professionnelle et familiale ne va pas de soi. En s'installant dans un lieu commun à la famille, les Mompreneurs sont également tenues de laisser à la famille ce qui est aussi son espace lorsque celle-ci est présente : avant d'avoir sa boutique et son laboratoire, Caroline, ancienne responsable de gestion en Île-de-France mariée à un cadre travaillant dans la logistique, s'est lancée dans les métiers de bouche en transformant «sa maison en fabrique ». Elle investit donc dans un équipement spécifique, comme « un four à échelle de refroidissement, un meuble qu' [elle a] fait fabriquer sur mesure pour la maison et puis [échange] les tables du salon [avec] les tables de marché qui se dépliaient dès [qu'elle] avai[t] beaucoup de volume à faire » ou transforme le bureau en « salle de stockage » et la salle d'eau en " chambre froide ». Non seulement, le « chez nous, c'était plus chez nous! », mais cette organisation spatiale de l'activité a également des incidences sur le rythme de travail :

« À partir de 5 heures et demie on range tout, on nettoie toute la table, on nettoie toute la cuisine, on empile les gâteaux pour pouvoir manger le soir, etc., on va chercher son fils, etc. et si les gâteaux sont pas finis, on redéballe tout à 21 heures et on finit à 23 heures. »

(Caroline, 33 ans, commerce de bouche, entretien du 16 juillet 2013)

Exerçant majoritairement à domicile, les Mompreneurs voient leur rythme de travail en subir les effets, à la fois dans sa continuité, mais aussi dans sa durée : alors que $61 \%$ des Mompreneurs installées chez elles travaillent au moins 30 heures par

20. À la différence de Frédérique, la situation économique du couple de Lili, qui suscite l'inquiétude de son conjoint, la contraint à maintenir une activité lui permettant notamment de participer au remboursement du prêt de leur appartement. 
semaine, c'est le cas de $79 \%$ de celles exerçant hors de leur domicile. Les répondantes à notre enquête quantitative ont dans leur ensemble des durées de travail hebdomadaires significativement moins importantes que celles des indépendants : $27 \%$ des Mompreneurs déclarent travailler plus de 40 heures par semaine ; $19 \%$ entre 35 et 40 heures, soit l'équivalent d'un temps plein ; $46 \%$ moins de 35 heures (temps partiel). Parmi ces dernières, $30 \%$ travaillent l'équivalent d'un mi-temps ou moins, $43 \%$ plus d'un mi-temps et moins d'un quatre-cinquièmes, $27 \%$ entre un quatre-cinquièmes et 35 heures. Les enquêtes Emploi du temps révèlent que la catégorie des indépendants en emploi est celle dont la durée de travail est la plus longue ( 6 h 51 contre 5 h 16 par jour en moyenne, soit près de 47 heures par semaine $)^{21}$; temps libre et temps domestique sont plus réduits, cette dernière variable étant à étudier en fonction du genre des indépendants $^{22}$. Prises dans cette réassignation à la sphère privée, les Mompreneurs semblent donc globalement moins se consacrer à leur temps professionnel que les indépendants dans leur ensemble.

\section{L'indépendance maternelle, entre risque économique et risque de déclassement}

Si les Mompreneurs privilégient la disponibilité auprès de leurs enfants et leur propre accomplissement comme marqueurs identitaires, elles n'en sont pas moins tenues par des considérations socio-économiques. Derrière leurs discours, nous tâcherons d'examiner plus avant les conséquences économiques de leurs parcours de Mompreneurs, en tentant d'y repérer différentes strates de « réussite ». L'examen au cas par cas révèle que ces strates sont masquées par une situation conjugale favorisée si bien qu'il est difficile de déterminer qui, au sein du couple, apporte davantage de revenus et de protections ${ }^{23}$. Comme les Mompreneurs ne sont pas épargnées par les négociations conjugales et les recompositions qui affectent les familles contemporaines, nous envisagerons dans un dernier temps les conséquences que la démobilisation de la famille peut engendrer pour la situation sociale et économique de ces femmes initialement plutôt favorisées et protégées.

\section{Des engagements concurrents qui grèvent les perspectives économiques des entreprises}

Dans le vaste mouvement de promotion de l'initiative économique individuelle et de l'entreprise de soi qui se déploie en France depuis la fin des années 1970, les médias

21. Source : Insee, enquête Emploi du temps 2009-2010.

22. Cf. l'article d'Amandine BARRoIS et François-Xavier DEVETTER (2017) dans ce dossier pour une investigation approfondie du temps de travail des indépendantes.

23. Nous n'avons pas eu accès au cours de l'enquête à un détail systématique des revenus des conjoints. Dans la plupart des cas toutefois, on devine que le conjoint est le principal apporteur de revenus. 
ont volontiers insisté sur l'accès aux responsabilités économiques que la création d'activités non salariées, comme celle des Mompreneurs, semble de prime abord représenter. Mais qu'en est-il réellement ? Pour évaluer sous un angle économique la catégorie des Mompreneurs, deux principes sont adoptés ici : nous nous demanderons d'abord si les activités créées permettent aux Mompreneurs de se dégager un revenu individuel (prenant en compte leurs éventuelles indemnités chômage) ; puis nous étudierons le niveau de ce revenu ainsi que les protections afférentes.

Les données quantitatives de notre enquête permettent de dresser plusieurs constats : sur les 268 répondantes, 194 déclarent un chiffre d'affaires annuel inférieur à 15000 euros. $72 \%$ de ces femmes dégagent ainsi un revenu mensuel a priori inférieur au salaire minimum interprofessionnel de croissance (Smic) mensuel brut à temps plein $\left(1480\right.$ euros $^{24}$ ). Parmi elles, 40 (soit $21 \%$ ) ont créé leur activité plus de deux ans avant l'enquête et ne peuvent plus dès lors toucher d'allocation-chômage (limitée à vingt-quatre mois pour les moins de 50 ans). 44 femmes (16\%) déclarent de leur côté un chiffre d'affaires annuel supérieur à 30000 euros. Entre ces deux pôles de revenus, seulement $10 \%$ des répondantes déclarent un chiffre d'affaires annuel oscillant entre un et deux Smic (hors soustraction des différentes charges relatives au fonctionnement de l'activité). Entre revenu d'appoint et relative aisance économique, il existe ainsi des situations économiques très contrastées entre les Mompreneurs, qui dépendent de différents facteurs, que les cas de Christelle et Marion permettent de mettre en lumière.

Fille d'un multi-entrepreneur et d'une mère cadre dans le secteur de la décoration, conjointe d'un spécialiste des flux financiers avec qui elle a eu deux enfants, Christelle occupe en région parisienne un poste de cadre issu de la promotion interne dans le secteur du papier qui la «passionne». Elle se voit refuser une promotion au motif qu'elle est à temps partiel et « explose »; toutefois, membre du comité d'hygiène et de sécurité des conditions de travail (CHSCT) de l'entreprise dans laquelle elle est employée depuis six ans environ, elle parvient à négocier une sortie en douceur. Décidée à créer un e-commerce familial dans le secteur du papier, dans lequel son conjoint alors au chômage travaillerait également, elle obtient en particulier une formation à la création et à la gestion de sites internet. Alors que son compagnon a retrouvé un emploi, Christelle poursuit le lancement de son entreprise depuis le garage réaménagé du pavillon dont ils sont propriétaires : notamment, elle s'affilie à une société coopérative de production (Scop) tout en conservant des liens étroits avec ses anciens clients et fournisseurs. Cette assise professionnelle maintenue est prolongée par la création d'une activité complémentaire dans le référencement de sites internet, activité pour laquelle elle a beaucoup travaillé et qu'elle a même fait certifier. En faisant fructifier les différentes ressources dont elle dispose (professionnelles, institutionnelles, organisationnelles et familiales), elle déclare un chiffre d'affaires de plus de 100000 euros annuels, ce que l'aisance qu'elle affiche (possession d'un $4 \times 4$ neuf, récit de nombreux voyages à l'étranger, etc.) confirme. Par ailleurs, lorsqu'elle se

24. Voir https://www.urssaf.fr/portail/home/taux-et-baremes/smic.html, consulté le 11 octobre 2017. 
lance dans la création de son activité, Christelle est déjà mère depuis quelques années et, si elle est largement à la manœuvre de l'ensemble de la sphère domestique, elle délègue une partie du travail domestique à une femme de ménage et partage avec son conjoint de nombreuses tâches parentales. La mobilisation de son conjoint, pour qui le projet d'entreprise était initialement pensé, participe ainsi également de sa réussite économique.

Marion, trentenaire, mère d'une petite fille, a quitté sa région d'origine pour s'établir dans le Sud de la France, loin de ses parents et tout particulièrement de son père, artisan. Son compagnon et elle rencontrent des difficultés pour trouver un emploi stable dans la région, qui est sévèrement touchée par le chômage. Après une première tentative de création d'activité en couple dans le secteur culturel, Marion, qui tombe enceinte, décroche un emploi en CDI dans une petite entreprise locale. Les relations sont toutefois extrêmement mauvaises au sein de l'entreprise et, même si elle devient déléguée du personnel avec une collègue dont elle se sent très proche, toutes deux peinent à résister aux pressions dont elles sont victimes. Décidées coûte que coûte à quitter l'entreprise, elles finissent par accepter une rupture conventionnelle négociée a minima. Prise par le travail parental dédié à sa fille et se disant passionnée depuis qu'elle est toute petite par le dessin, Marion décide avec son amie d'éditer à compte d'auteurs deux ouvrages de littérature enfantine ; une agence de Pôle emploi les oriente alors vers une couveuse d'entreprise spécialisée dans l'art, qui leur fournit une structure juridique pour asseoir le projet et un accompagnement technique, en particulier sur le plan comptable. Les deux amies, qui vivent de leurs prestations chômage, financent à hauteur de 3000 euros deux albums sur leurs deniers personnels, sans passer par un emprunt. Un an et demi après le démarrage de leur activité, elles ont écoulé 400 exemplaires (dont 300 pour le premier album) sur les 2000 édités et doivent encore gagner 1000 euros pour rembourser leur mise de départ. Le projet est ainsi un échec sur le plan économique, si bien que lorsque leur contrat avec la couveuse s'arrête en novembre 2012, il ne leur est pas possible d'envisager une immatriculation autonome de leur activité. Dans le même temps, Marion atteint deux ans de chômage, ses indemnités s'arrêtent début 2013, la contraignant à retrouver d'urgence un revenu. Au cours de l'entretien, elle précise que, parallèlement à ce projet non salarié qu'elle met très largement en avant, elle a continué à chercher un emploi salarié, sans parvenir à décrocher un seul contrat compte tenu du nombre d'offres restreint sur la région. Son entourage cherche à l'aider : sa tante par exemple utilise son réseau pour lui trouver quelques contrats d'infographiste. Pour pouvoir facturer, Marion s'immatricule en tant qu'auto-entrepreneuse en janvier 2013 mais son activité reste limitée (elle dit ainsi ne quasiment pas travailler pendant les trois premiers mois de l'année) et ses contrats ne sont décrochés que par l'entremise de son entourage personnel (« ça se débloque entre avril et mai, surtout par connaissances, des amis, de la famille, des amis de la famille »). Elle considère le mois précédant notre rencontre, c'est-à-dire mai 2013, comme son meilleur mois car elle est parvenue à se dégager « un salaire correct » : 
«Entre le peu que je me suis remboursée de l'asso, la mission que j'ai fait[e] et les petits contrats à côté, en net, j' ai dû me faire 1400 euros, ce qui pour moi était bien en démarrage d'activité, parce que ça fait très peu de temps que je me suis lancée. »

(Marion, 32 ans, auto-entreprise de services aux entreprises, entretien du 17 juin 2013)

Marion ne bénéficie pas des mêmes ressources que Christelle ; elle ne peut pas compter sur le soutien financier de ses proches. Certes, un héritage familial important a permis à son conjoint d'acheter comptant le pavillon dans lequel la famille réside ; en revanche, la situation professionnelle de Marion reste précaire.

On trouve ainsi chez les Mompreneurs des situations hétérogènes, allant d'un confort objectif garanti par des ressources nombreuses intensément mises à profit à des cas nettement plus précaires sur le plan individuel. Cette hétérogénéité reste toutefois masquée par la vie de couple : Marion estime par exemple qu'elle peut vivre confortablement avec 1 000-1 500 euros mensuels parce qu'elle bénéficie du revenu et du patrimoine de son conjoint. Mais que se passe-t-il quand le cadre familial ne soutient plus ces femmes?

\section{Quand la famille ne fait plus « cause commune ${ }^{25}$ »}

Conduire une enquête au long cours a permis de voir évoluer, dans un sens positif ou négatif, la situation des Mompreneurs rencontrées. Plusieurs témoignent d'un d'échec, qui peut prendre des formes variées. Il arrive d'abord que l'activité indépendante s'arrête : certaines Mompreneurs font faillite, en particulier celles qui se lancent dans une activité commerciale et qui se retrouvent avec des stocks non liquidés et des fournisseurs qu'il devient difficile de payer. C'est ce qui est arrivé à Delphine, mariée avec un agent d'une collectivité territoriale et ancienne cadre dans une société de services. Après son licenciement, elle crée une société à responsabilité limitée (SARL) dans le secteur de la parapharmacie : parallèlement à son site d'e-commerce, elle installe dans le pavillon familial une pièce où elle espère organiser des ateliers olfactifs. Un an et demi après notre première rencontre, elle confie que l'activité n'a pas rencontré le succès escompté, ce qui a considérablement inquiété son conjoint : aussi a-t-elle, en réponse à ses pressions, pris un emploi de vendeuse à temps partiel dans un magasin bio à proximité de chez elle. Elle explique s'être résolue à liquider son activité et s'être mise en recherche d'un nouvel emploi salarié : après plusieurs refus pour des postes de cadre, elle finit par accepter un poste d'assistante commerciale à quatre cinquièmes dans une très petite entreprise locale.

Si Delphine ne bénéficie plus des pleines protections de l'emploi salarié, elle reste protégée par sa situation familiale, malgré les difficultés conjugales dont elle a pudiquement fait part. Dans d'autres cas en revanche, la famille se recompose et c'est la liberté même de ces femmes qui est mise en question, ce que laisse entrevoir

25. Voir Roy, 2005. 
le cas de Barbara, proche de la cinquantaine et mère d'un garçon de 8 ans. Fille d'un ingénieur et d'une femme au foyer, danseuse amatrice, elle tente de s'insérer sans succès dans le monde de la danse. Elle occupe ensuite des postes d'hôtesse d'accueil dans différentes maisons de couture, jusqu'à sa rencontre avec un chef d'entreprise installé dans le sud de la France. Elle devient sa compagne et collaboratrice pendant plusieurs années, sans aucun statut conjugal ni professionnel pour asseoir sa situation : à son décès, elle bascule dans une grande précarité et vit quelques années du revenu de solidarité active (RSA). Puis elle rencontre le " père de son fils », alors informaticien indépendant. Elle glisse au cours de l'entretien que l'enfant est un « miracle » : à plus de 40 ans, mais souhaitant vivement devenir mère, elle décide de faire un enfant tout en sachant que « son couple n'irait pas très loin ». À la naissance de l'enfant, le couple est déjà séparé mais cohabite dans une maison dont son ex-compagnon est propriétaire. Barbara dit vivre comme une « bourgeoise au foyer » pendant plusieurs années : tout en s'occupant de son fils, elle suit des cours et diverses formations dans le champ de la psychologie. Elle présente avec beaucoup de sérénité la relation qu'elle entretient avec le père de son fils, avec qui elle partage la maison, mais aussi les comptes en banque :

« Un jour il m'a commandé une carte bleue sur son compte et il m'a donné sa carte bleue et depuis je n'ai jamais rendu cette carte bleue, qui ne m'a jamais été demandée $[\ldots]$ et puis il y a quelque chose de, je sais pas de naturel, oui, et encore plus depuis que je suis devenue maman. En fait, c'est comme si c'était mon rôle et mon salaire, de m'occuper qu'il y ait à manger à la maison, de m'occuper de mon fils, qu'il soit bien élevé [...]. Et puis aussi, c'est parce qu'il me fait totalement confiance et qu'il peut le faire. »

(Barbara, 48 ans, auto-entreprise de services aux particuliers, entretien du 11 juin 2013)

Elle dit ainsi apprécier le « confort » de sa situation, la question financière n'étant «pas son problème », mais plusieurs éléments ternissent ce tableau : ses parents sont angoissés car ils jugent qu'elle se trouve dans « une précarité effrayante »; de plus, elle rencontre des difficultés pour trouver un nouveau compagnon (ses rencontres trouvent sa situation « trop space ») et semble très isolée dans l'éducation de son fils, dont le père semble peu s'occuper au quotidien. Barbara a sinon lancé une activité de coaching sous le régime de l'auto-entrepreneur, qu'elle complète avec des cours de gym. Elle espère que le développement de cette double activité lui permettra de prendre dans quelques mois un appartement, signe de son souhait de sortir d'une situation au confort finalement tout relatif. Si le cas de Barbara est à de nombreux égards atypique, il rend compte de la dépendance conjugale des Mompreneurs qui exercent une activité indépendante peu - voire pas - rémunératrice : rappelons que $72 \%$ de ces femmes dégagent moins d'un Smic mensuel à temps plein. La quête d'accomplissement de soi dans laquelle elles se sont lancées incite en outre une part importante d'entre elles, parmi les moins dotées et les plus éloignées au départ du monde de l'indépendance, à privilégier des activités certes valorisantes mais moins lucratives que des activités salariées permettant un revenu régulier. Sous la création d'entreprise se cache donc un 
déclassement économique individuel important, que la figure de l'entrepreneur-euse masque. En cas de divorce, elles sont extrêmement dépendantes du maintien d'une solidarité financière entre les ex-conjoints, dont on sait qu'elle ne parvient jamais à compenser le confort assuré par le couple (BONNET et al., 2015). Enfin, à plus long terme, ces femmes voient leur niveau de protection sociale, notamment en matière de retraite, obéré par la faiblesse de leur chiffre d'affaires ${ }^{26}$. Sous le couvert d'une entreprise globale de soi, une hiérarchie genrée classique reste ainsi à l'œuvre et semble même s'accentuer. Entre travail, marché et famille, la promesse de responsabilisation et de conciliation des Mompreneurs relève bien plus d'un mirage que d'un miracle, $y$ compris pour des femmes qui bénéficiaient pourtant de ressources solides pour atténuer leur domination au sein des rapports sociaux de sexe.

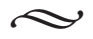

Au terme de cette analyse, il apparaît que les Mompreneurs font avant tout de leur entrée dans l'indépendance un vecteur identitaire, qui leur permet d'exercer une activité plus expressive qu'instrumentale mais également de développer un rapport lui aussi identitaire au travail parental. Elles sont toutefois fortement réassignées au foyer et ce n'est que parce qu'elles parviennent à mobiliser activement les ressources, nombreuses et bien ajustées, dont elles disposent initialement, que certaines parviennent à s'assurer une relative stabilité individuelle tandis que d'autres se trouvent entièrement dépendantes de leur conjoint. Globalement, pour celles qui ne réussissent pas à faire décoller leur activité et à se garantir un revenu, leur parcours favorise un déclassement social non négligeable; et celles qui voient en plus le soutien familial et conjugal s'altérer sont exposées à un risque de déclassement économique et social à plus long terme.

Aussi ce phénomène interroge-t-il plusieurs faits à la croisée du travail, de la famille et des politiques publiques qui les concernent : l'intensification des normes parentales, qui s'adressent encore largement aux mères, interroge leur capacité à tenir dans un travail requérant par ailleurs toujours plus de performance. La création d'entreprise, par l'apparente flexibilité horaire qu'elle promet, peut ainsi être utilisée comme un dispositif inattendu d'articulation des temps de vie. Néanmoins, nous l'avons vu à travers les parcours des Mompreneurs, travail professionnel et travail parental, même exercés dans le même espace, sont en réalité plus juxtaposables que superposables. Les Mompreneurs interrogent également avec acuité les dispositifs publics d'activation, tels que ceux dédiés à la création d'entreprise, qui demandent une très forte mobilisation de l'ensemble des ressources dont un individu peut disposer : au sein de ce groupe social relativement favorisé, ce ne sont pas seulement les

26. Rappelons en effet que, si la protection sociale des indépendant·e·s s'est progressivement alignée sur celle des salarié·e·s, un chiffre d'affaires minimal est toutefois requis pour valider des trimestres. Aussi faut-il par exemple, en 2017, dégager 4190 euros de chiffre d'affaires pour une activité de services pour valider deux trimestres (source : https://www.rsi.fr/cotisations/ae/protection-sociale.html, consulté le 6 octobre 2017). 
ressources collectives, comme les aides financières à la création d'entreprise ou l'offre de formation professionnelle, qui sont mises à l'épreuve, mais également les ressources biographiques d'un individu prêt à s' assumer comme tel. S'agissant spécifiquement des femmes, l'étude des Mompreneurs rappelle que dans les rapports sociaux de sexe, elles restent maintenues dans une position infériorisée, qui structure leur rapport au travail, est réactualisée par le travail parental et légitimée par des normes de la parentalité toujours conjuguée au féminin. Leur capacité à l'individuation et à l'émancipation est dès lors d'autant plus difficile à mettre en œuvre, y compris pour les plus favorisées d'entre elles.

\section{BibliogRAPHIE}

ABDelnour S. (2013), «L'entrepreneuriat au service des politiques sociales : la fabrication du consensus politique sur le dispositif de l'auto-entrepreneur », Sociétés contemporaines, $\mathrm{n}^{\mathrm{o}}$ 89, pp. 131-154.

AнL H. (2004), The Scientific Reproduction of Gender Inequality, a Discourses Analysis of Research Texts on Women's Entrepreneurship, Copenhague, Copenhagen Business School Press.

BARRoIs A., DeVETTER F.-X. (2017), « Femmes salariées et non salariées : quelles différences de temps de travail ? », Travail et Emploi, $\mathrm{n}^{\circ} 150$, pp. 101-130.

Berta N., Signoretto C., VAlentin J. (2012), « La rupture conventionnelle : objectifs officiels versus enjeux implicites? », Revue française de socio-économie, $\mathrm{n}^{\circ}$ 9, pp. 191-208.

Bonnet C., Garbinti B., Solaz A. (2015), «Les conditions de vie des enfants après le divorce », Insee première, $\mathrm{n}^{\circ} 1536$.

Bourdieu P. (1979), La Distinction. Critique sociale du jugement, Paris, Éditions de Minuit.

Dalmasso R., Gomel B., Méda D., Serverin É. (2012), « Des ruptures conventionnelles vues par des salariés. Analyse d'un échantillon de cent une ruptures conventionnelles signées fin $2010 »$, Rapport de recherche, $\mathrm{n}^{\circ}$ 80, Noisy-le-Grand, Centre d'études de l'emploi.

DARBUS F. (2008), « L'accompagnement à la création d'entreprise. Auto-emploi et recomposition de la condition salariale », Actes de la recherche en sciences sociales, $\mathrm{n}^{\circ} 175$, pp. 18-33.

DelPhy C. (1998), L'Ennemi principal, tomes 1 et 2, Paris, Syllepse.

Delphy C. (2015), Pour une théorie générale de l'exploitation. Des différentes formes d'extorsion de travail aujourd'hui, Paris, Syllepse.

Dujarier M.-A. (2006), L'Idéal au travail, Paris, Presses universitaires de France.

Ehrenberg A. (1991), Le Culte de la performance, Paris, Calmann-Lévy.

Fouquet A. (2005), «Les femmes chefs d'entreprise : le cas français », Travail, genre et sociétés, $\mathrm{n}^{\circ} 13$, pp. 31-50. 
GARCIA S. (2011), Mères sous influence : de la cause des femmes à la cause des enfants, Paris, La Découverte.

HaYs S. (1996), The Cultural Contradictions of Motherhood, New Haven, London, Yale University Press.

Jourdain A. (2017), « Des artisans d'art aux artisanes d'art. Ce que le genre fait aux métiers d'art indépendants », Travail et Emploi, $\mathrm{n}^{\mathrm{o}}$ 150, pp. 25-52.

LANDOUR J. (2015a), « Quand les mères deviennent entrepreneurs. Image et expériences des "Mompreneurs" ", Sociétés contemporaines, n ${ }^{\circ}$ 98, pp. 137-168.

LANDOUR J. (2015b), « Les collectifs de "Mompreneurs", une solidarité professionnellement porteuse ? », Modern \& Contemporary France, vol. 23, n 4, pp. 457-473.

LANDOUR J. (2016), « La parentalité, une contribution au capital des femmes des classes supérieures ?», Genre, sexualité et société [en ligne], n ${ }^{\circ} 16$ : https://gss.revues.org/3886, consulté le 12 octobre 2017.

Maruani M., Meron M. (2012), Un siècle de travail des femmes en France (1901-2011), Paris, La Découverte.

Meurs D., Pailhé A., Ponthieux S. (2010), «Enfants, interruptions d'activité des femmes et écart de salaire entre les sexes », Revue de l'OFCE, n 114, pp. 113-133.

Osty F. (2003), Le Désir de métier. Engagement, identité et reconnaissance au travail, Rennes, Presses universitaires de Rennes.

Pailhé A., Solaz A. (2006), «Vie professionnelle et naissance : la charge de la conciliation repose essentiellement sur les femmes », Population et sociétés, $\mathrm{n}^{\circ} 426$.

Pailhé A., Solaz A. (2012), « Durée et conditions de retour à l'emploi des mères après une naissance », Retraite et société, $\mathrm{n}^{\circ}$ 63, pp. 51-77.

RABIER M. (2013), Entrepreneuses de cause. Contribution à une sociologie des engagements des dirigeantes économiques en France, thèse de doctorat en sociologie, École des hautes études en sciences sociales.

RÉGNIER-LOILIER A. (2009), «L'arrivée d'un enfant modifie-t-elle la répartition des tâches domestiques au sein du couple ? », Population et sociétés, $\mathrm{n}^{\circ} 461$.

Roy D. (2005), « Tout ce qui est à moi est à toi ? Mise en commun des revenus et transferts d'argent dans le couple », Terrain, n $^{\circ} 45$, pp. 41-52. 